\title{
Tunable Chromatic Dispersion Management of Optical Fibre Communication Link using SOA
}

\author{
Md Shakil Ahmed \\ Electronic and Electrical Engineering Dept. \\ University of Strathclyde \\ Glasgow, United Kingdom \\ shakil.ahmed@strath.ac.uk
}

\author{
Ivan Glesk \\ Electronic and Electrical Engineering Dept. \\ University of Strathclyde \\ Glasgow, United Kingdom \\ ivan.glesk@strath.ac.uk
}

\begin{abstract}
High data rate communication demands the well compensated chromatic dispersion in the optical fibre communication. Generally dispersion compensated fibre (DCF) modules are utilized in the link with known fibre lengths. On the other hand, at ultra high data rates when a new user wants to access the network with a connecting cable of unknown length, automated dispersion compensation technique will ensure the error free communication with much greater flexibility to operate optical networks. An experiment was carried out to compensate the chromatic dispersion in an optical fibre network by passing the received data signal through an SOA. By controlling the SOA pump current, it was possible to easily compensate the chromatic dispersion without changing the lengths of DCF.
\end{abstract}

Keywords-dispersion; compensation; pulsewidth; chirp; optical fibre

\section{INTRODUCTION}

In high speed optical communication, chromatic dispersion is an inherent problem. The term dispersion is defined as a pulse spreading in an optical system. When a pulse of light passes through a fibre optic cable, the aperture, core diameter, wavelength, refractive index profile, laser linewidth properties etc. cause the optical pulse broadening [1]. This could be an immense drawback in optical communication. As the optical bandwidth gradually increases, the inter-bit distances also become smaller. It is well known that in the standard SMF-28 fiber with the increase of cable length, due to chromatic dispersion (CD), the data signal pulse width increases by a rate of $16 \mathrm{ps} / \mathrm{km}$. As a result, there is possibility for bits overlapping at the receiver end and will become difficult to distinguish between ' $1 \mathrm{~s}$ ' and ' $0 \mathrm{~s}$ '. This phenomenon is commonly known as inter symbol interference (ISI). This could be a big issue for a network traffic management. Network operators do not normally know the exact length of fibre cables and as such the $\mathrm{CD}$ value is also not up front known. For that reason, operators need to keep a large number of DCF modules for the necessary dispersion compensation of communication links. But for the data rates of more than $40 \mathrm{~Gb} / \mathrm{s}$, the threshold for accumulated dispersion is about 16 times smaller than that of $10 \mathrm{~Gb} / \mathrm{s}$. Because, when a bit rate increases by a factor of four, then the $\mathrm{CD}$ effect increases by a factor of 16 [2]. The important issue is this, if the compensating value does not matches a certain percentage of allowable $\mathrm{CD}$, then the link may not operate at all due to ISI effects. Therefore, a preferable solution would be to develop an automatic dispersion monitoring [3] and compensating system $[4,5,6]$ so that higher bandwidth requirements might be fulfilled without any occurrence of ISI effects without usage of additional DCF. A good number of researches presented different techniques of dispersion compensation. Dispersion compensation using AWG, FBG are important to note $[7,8]$. Another technique of extending the signal transport distance of fiber optics link using the chirp control in SOA was shown in [9]. In this paper, the tunable chromatic dispersion management by SOA with changing SOA driving current will be described and experimentally demonstrated.

\section{THEORETICAL BACKGROUND}

By varying the SOA drive current i.e., the SOA gain, the carrier density inside of the SOA varies; as a result the refractive index of SOA also changes which subsequently broadens or compresses the passing signal pulse-width [10]. This is a result of changes of a real and imaginary part of a dielectric constant of the SOA (its waveguide medium) which are related by Kramers-Kronig (KK) dispersion relations [11]. The complex refractive index of SOA can be represented by:

$$
n=n_{0}+\Delta n^{\prime}+\mathrm{i} \Delta n^{\prime}
$$

Here, $\Delta n^{\prime}+\mathrm{i} \Delta n^{\prime}$ represent the real and imaginary part of refractive index changes due to the carrier density changes by current injection; $n_{0}$ is the real part of the SOA refractive index. Considering $n_{0}$ as a constant, through Kramers-Kronig (KK) relation, we can mathematically show [11]:

$$
\begin{aligned}
\Delta n^{\prime}(E) & =\frac{2}{\pi} P \int_{0}^{\infty} \frac{E^{\prime} \Delta n^{\prime \prime}\left(E^{\prime}\right) d E^{\prime}}{E^{\prime 2}-E^{2}}= \\
& =-\frac{1}{2 \pi^{2}} P \int_{0}^{\infty} \frac{\Delta g\left(E^{\prime}\right) d E^{\prime}}{{E^{\prime}}^{2}-E^{2}}
\end{aligned}
$$

Here, $P$ denotes the principal value integral [12] and $\Delta g$ is a gain change.

From the above Eq. (2) we can find a relationship between SOA gain changes which then effect the refractive index changes of the SOA and thereby result in compression or expansion of optical pulse which passes through the SOA. 


\section{EXPERIMENTAL RESULTS}

In our experiment we used a testbed setup in Fig. 1 comprising of picosecond (ps) mode locked (ML) laser, erbium-doped fiber amplifier (EDFA), SOA, and a $17 \mathrm{~km}$ long SMF-28 optical fiber link between University of Strathclyde and University of Glasgow which is dispersion compensated by using DCF. A fibre Brag grating (FBG) encoder generates four wavelengths $\left(\lambda_{1}=1551.72 \mathrm{~nm}, \lambda_{2}=\right.$ $1550.92 \mathrm{~nm}, \lambda_{3}=1552.52 \mathrm{~nm}, \lambda_{4}=1550.12 \mathrm{~nm}$ ) by slicing the optical supercontinuum.

In our experiment an add/drop AWG module is used to select a single wavelength $\lambda_{2}$ which is then transmitted down the optical fiber. The compensated optical data pulses having $\mathrm{FWHM}=23$ ps first pass a bandpass filter (BPF) and then enter an SOA for a final fine dispersion tuning. By varying the SOA drive current, we controlled the chromatic dispersion compensation i.e., optical pulse compression or expansion.

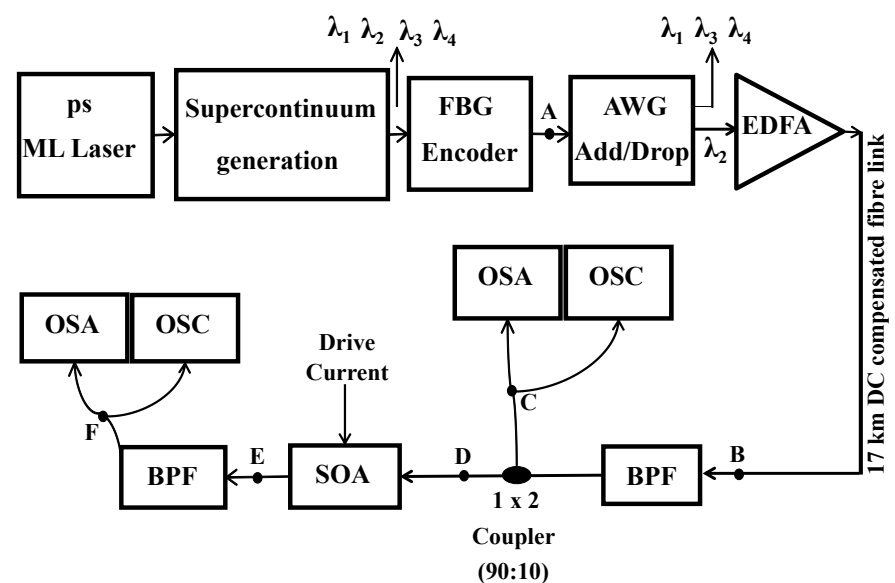

Fig. 1. Experimental setup for a chromatic dispersion compensation in optical fiber by use of SOA.

\section{OBTAIND RESULTS}

At the input of SOA (point D), the measured pulsewidth of the data pulse was 23 ps (Fig. 2). By adjusting the SOA drive

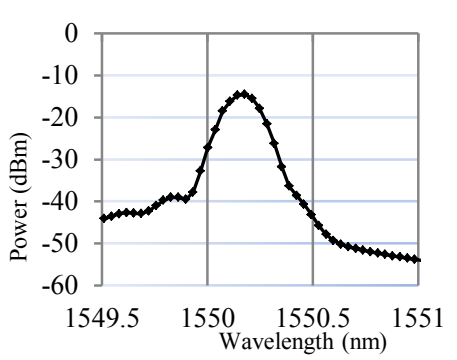

(a)

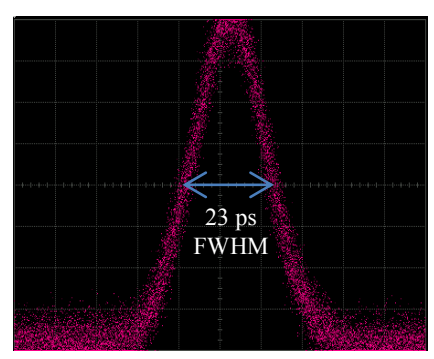

(b)
Fig. 2. (a) Frequency Domain representation of returned data at the SOA input (b) Time Domain representation of returned data at the SOA input

current was found that the pulsewidth can be compressed to $20 \mathrm{ps}$ for the SOA drive current of $29 \mathrm{~mA}$ (Fig. 3). After changing the SOA drive current to $70 \mathrm{~mA}$ (Fig. 4), the pulsewidth of the data signal has reached again 23 ps (the same value of input data). But at this condition, the input signal has been amplified to $-10 \mathrm{dBm}$ level. This is an added advantage of this technique since it can also alter the received signal power level to compensate for the insertion loss in optical network [9]. At the SOA drive current of $70 \mathrm{~mA}$ there was no compression or expansion just a gain change of the passing signal. After further increase in SOA drive current to $150 \mathrm{~mA}$, the pulse-width was expanded to 25 ps (Fig. 5).

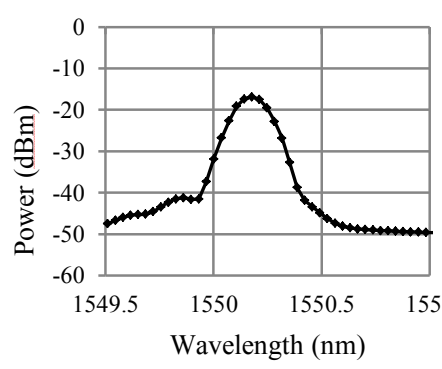

(a)

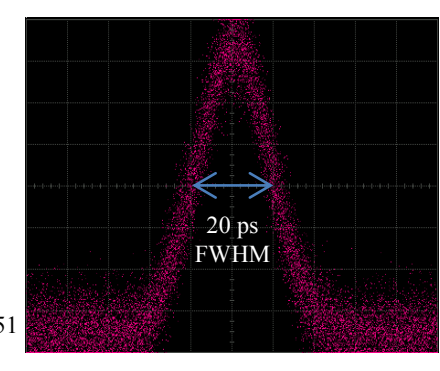

(b)
Fig. 3. (a) Frequency Domain representation of the compressed data pulse at the output for $29 \mathrm{~mA}$ SOA drive current (b) Time Domain representation of the compressed data at the output for $29 \mathrm{~mA}$ SOA drive current

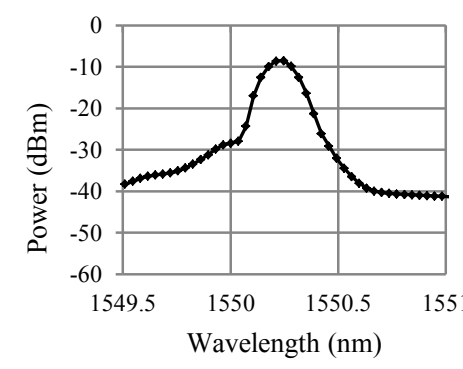

(a)

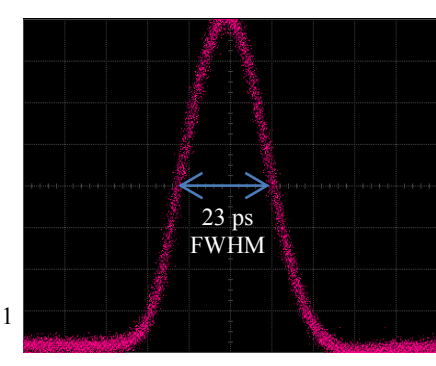

(b)
Fig. 4. (a) Frequency Domain representation of the data pulse at the output for $70 \mathrm{~mA}$ SOA drive current (b) Time Domain representation of the data at the output for $70 \mathrm{~mA}$ SOA drive current

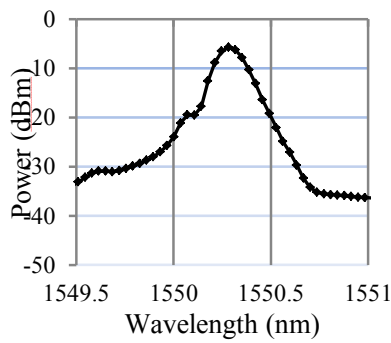

(a)

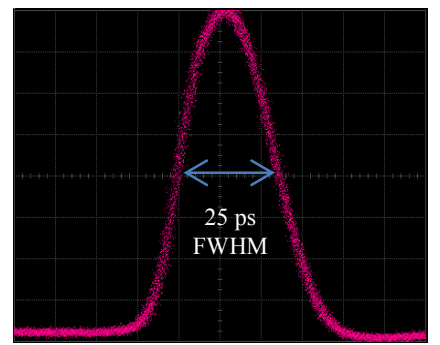

(b)
Fig. 5. (a) Frequency Domain representation of the broadened data pulse at the output for $150 \mathrm{~mA}$ SOA drive current (b) Time Domain Representation of the broadened data pulse at the output for $150 \mathrm{~mA}$ SOA drive current

The graph showing the SOA gain vs SOA drive current is shown in Fig. 6a. The changes of output/input pulse width ratio vs SOA gain are shown in Fig. 6b. From Fig. 6a, it is seen that SOA gain saturates at the drive current of $\sim 150 \mathrm{~mA}$. Below SOA gain of 5 is the pulse compression region and above SOA gain of 5 is the data pulse expansion region (see Fig. 6b). The SOA gain of 5 is equivalent to SOA drive current of $\sim 70 \mathrm{~mA}$. 


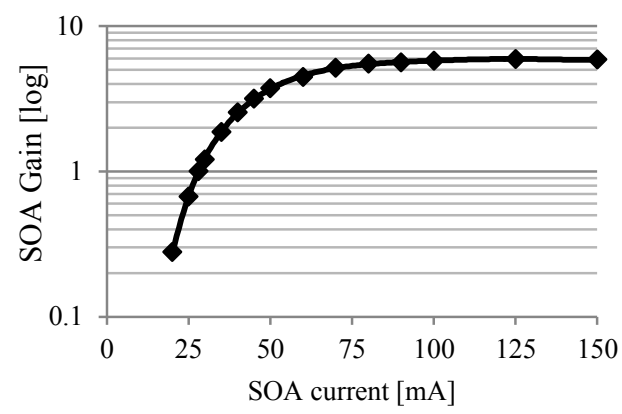

(a)

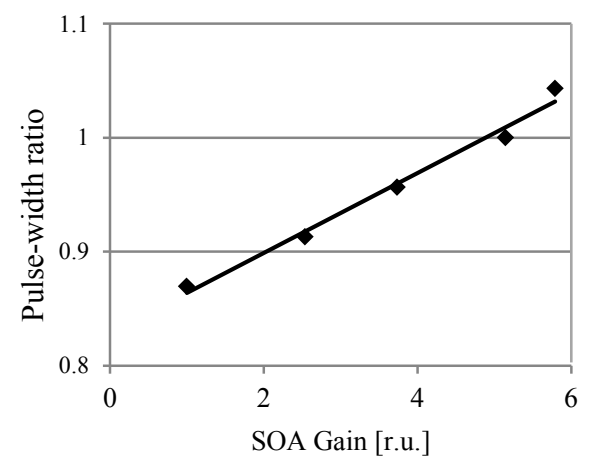

(b)

Fig. 6a. SOA Gain vs Drive Current, (b) output/input pulse width ratio vs SOA gain

\section{DISCUSSION}

It is inportant to note that both, pulse compression and expansion are possible by varying the SOA drive current. This is important feature for fine tuning of optical fiber dispersion and achieving compensation or expansion of optical pulses traveling in optical newtworks. This will enable us to fine tune chromatically dispersion compensated fiber optic networks for sudden small changes of dispersion. This simple approach demonstrates that by controlling the SOA drive current, a pulse compression or expansion can be achieved. This gives a great operational flexibility to network operators for managing high-speed optical communication systems to mitigate detremental efects of dispersion.

In this experiment, the SOA drive current of $70 \mathrm{~mA}$ may be considered as the "neutral" setting point where there is neither compensation nor expansion of optical signal data pulses. By increasing or decresing the drive current around this value, we can expand or compress the signal pulse width. In such a way, we can fulfill the practical requirement of chromatic dispersion fine tuning needs in high speed communication networks.

\section{CONCLUSION}

Continuous chromatic dispersion compensation in optical fiber network is import for achieving flexibility of data communication at ultra-high speeds of transmissions. The chromatic dispersion effect in optical fibers becomes severe for ultra-high data rates and/or for greater transmission distances in optical networks. The conventional method of chromatic dispersion compensation are often using matching lengths of DCF modules which is tedious and time consuming and becomes difficult to implement if the fiber lengths are not known before head or dispersion is changing due to environmental changing conditions. We have demonstrated chromatic dispersion compensation technique in SME-28 optical fiber using an SOA by varying its drive current. This method for the chromatic dispersion compensation was tested in $17 \mathrm{~km}$ long optical fiber testbed. We have shown both, pulse compensation and expansion between 20 ps to 25 ps of the original 23 ps optical pulse. This method is simple the flexibility of achieving either compression or expansion of data pulse width.

\section{References}

[1] Massa, N., 2000. Fiber optic telecommunication. Fundamentals of Photonics. University of Conneticut.

[2] Willner, A.E. and Hoanca, B., 2002. Fixed and tunable management of fiber chromatic dispersion. Optical Fiber Telecommunications IVB, pp.642-724.

[3] Pan, Z., Yu, C. and Willner, A.E., 2010. Optical performance monitoring for the next generation optical communication networks. Optical Fiber Technology, 16(1), pp.20-45.

[4] Pan, Z., Yu, Q., Xie, Y., Havstad, S.A., Willner, A.E., Starodubov, D.S. and Feinberg, J., 2001, March. Chromatic dispersion monitoring and automated compensation for NRZ and RZ data using clock regeneration and fading without adding signaling. In Optical Fiber Communication Conference (p. WH5). Optical Society of America

[5] Petersen, M.N., Pan, Z., Lee, S., Havstad, S.A. and Willner, A.E., 2002. Online chromatic dispersion monitoring and compensation using a single inband subcarrier tone. Photonics Technology Letters, IEEE, 14(4), pp.570-572.

[6] A. Sano, T. Kataoka, M. Tomizawa, K. Hagimoto, K. Sato, K. Wakita, and K. Kato, "Automatic dispersion equalization by monitoring extracted-clock power in a $40-\mathrm{Gbit} / \mathrm{s}, 200-\mathrm{km}$ transmission line," in ECOC, 1996, TuD.3.5, pp. 2207-2210.

[7] Kerbstadt, F. and Petermann, K., 2005. Analysis of adaptive dispersion compensators with double-AWG structures. Journal of lightwave technology, 23(3), p.1468.

[8] Pan, Z., Song, Y.W., Yu, C., Wang, Y., Yu, Q., Popelek, J., Li, H., Li, Y. and Willner, A.E., 2002. Tunable chromatic dispersion compensation in $40-\mathrm{Gb} / \mathrm{s}$ systems using nonlinearly chirped fiber Bragg gratings. Journal of lightwave technology, 20(12), p.2239.

[9] T.Watanabe et al.: Transmission Performance of Chirp-Controlled Signal by Using Semiconductor Optical Amplifier. IEEE Journal of Lightwave Technology (August 2000), 1069-1077

[10] Agrawal, G.P. and Olsson, N.A., 1989. Amplification and compression of weak picosecond optical pulses by using semiconductor-laser amplifiers. Optics letters, 14(10), pp.500-502.

[11] Henry, C.H., Logan, R.A. and Bertness, K.A., 1981. Spectral dependence of the change in refractive index due to carrier injection in GaAs lasers. Journal of Applied Physics, 52(7), pp.4457-4461.

[12] Landau, D. and Lifshitz, E.M., 1979. Electrodynamics o/Continuous Media (Permagon, New York, 1960). In $s F$. B. Foote and DT Hodges, in Proceedingso/the Fourth International Conference on In/rared and Millimeter Waves and Their Application, Florida (pp. 78-9). 